\title{
On the Metric Dimension of the Reduced Power Graph of a Finite Group
}

\author{
Xuanlong $\mathrm{Ma}^{*}$ and Lan Li
}

\begin{abstract}
Let $G$ be a finite group. The reduced power graph of $G$ is the undirected graph whose vertex set is $G$, and two distinct vertices $x$ and $y$ are adjacent if $\langle x\rangle \subset\langle y\rangle$ or $\langle y\rangle \subset\langle x\rangle$. In this paper, we give tight upper and lower bounds for the metric dimension of the reduced power graph of a finite group. As applications, we compute the metric dimension of the reduced power graph of a $\mathcal{P}$-group, a cyclic group, a dihedral group, a generalized quaternion group, and a group of odd order.
\end{abstract}

\section{Introduction}

All graphs considered in this paper are finite, undirected, with no loops and no multiple edges. Let $\Gamma$ be a graph. The vertex set of $\Gamma$ is denoted by $V(\Gamma)$. Let $x, y \in V(\Gamma)$. The distance between $x$ and $y$ in $\Gamma$ is the length of a shortest path from $x$ to $y$ and is denoted by $d_{\Gamma}(x, y)$. If the situation is unambiguous, we denote $d_{\Gamma}(x, y)$ simply by $d(x, y)$. If there exists $z \in V(\Gamma)$ such that $d(x, z) \neq d(y, z)$, then we say that $z$ resolves vertices $x$ and $y$. A subset $S$ of $V(\Gamma)$ is called a resolving set of $\Gamma$ if every pair of distinct vertices of $\Gamma$ is resolved by some vertex of $S$. The metric dimension of $\Gamma$, denoted by $\operatorname{dim}(\Gamma)$, is the minimum cardinality of a resolving set of $\Gamma$. In the 1970s, the metric dimension of a graph was introduced independently by Harary and Melter [12] and Slater [26]. It was noted in [10 that determining the metric dimension of a graph is an NP-complete problem.

Graphs associated with groups and other algebraic structures have been actively investigated, since they have valuable applications (cf. [15, 19]) and are related to automata theory (cf. [16, 17]). The undirected power graph $\mathcal{P}(G)$ of a finite group $G$ has vertex set $G$ and two distinct elements are adjacent if one is a power of the other. The concepts of power graph and undirected power graph were first introduced by Kelarev and Quinn 18 and Chakrabarty et al. [6], respectively. In recent years, the study of power graphs has been growing, see, for example, [4, 5, 21, 22]. Also, see [1] for a survey of results and open problems on power graphs.

With an aim to avoid the complexity of edges in power graphs, Rajkumar and Anitha 23 introduced the reduced power graph $\mathcal{P}_{R}(G)$ of a group $G$, which is an undirected

Received September 21, 2020; Accepted September 15, 2021.

Communicated by Sen-Peng Eu.

2020 Mathematics Subject Classification. 05C25, 05C12.

Key words and phrases. reduced power graph, metric dimension, finite group.

*Corresponding author. 
graph with vertex set $G$, and two distinct vertices $x, y$ are adjacent if $\langle x\rangle \subset\langle y\rangle$ or $\langle y\rangle \subset\langle x\rangle$. In other words, $\mathcal{P}_{R}(G)$ is the subgraph of $\mathcal{P}(G)$ obtained by deleting all edges $\{x, y\}$ with $\langle x\rangle=\langle y\rangle$, where $x$ and $y$ are distinct elements of $G$. In [23], the authors studied the interplay between the algebraic properties of a group and the graph theoretic properties of its reduced power graph. Recently, Anitha and Rajkumar 3] characterized the groups with planar, toroidal and projective planar reduced power graphs, and they also determined the Laplacian spectrum of the reduced power graph of some finite groups [24]. Ma 20] characterized the proper connection number of a reduced power graph. Moreover, see [2,25] for some more properties of this graph.

The metric dimension of a power graph was studied in [9]. Here, we study the metric dimension of a reduced power graph. Specifically speaking, we give tight upper and lower bounds for the metric dimension of the reduced power graph of a finite group (see Theorem 3.2. As applications, we compute the metric dimension of the reduced power graph of a $\mathcal{P}$-group, a cyclic group, a dihedral group and a generalized quaternion group.

\section{Preliminaries}

This section introduces some basic definitions and notations that are used throughout the paper, and proves some technical lemmas required for the proofs of our main results.

All groups considered in this paper are finite. We always use $G$ to denote a finite group, and use $e$ denote the identity element of $G$. The order of an element $x$ of $G$, denoted by $o(x)$, is defined as the cardinality of the cyclic subgroup $\langle x\rangle$. An element of order 2 is called an involution. A maximal cyclic subgroup of $G$ is a cyclic subgroup, which is not a proper subgroup of some cyclic subgroup of $G$. The set of all maximal cyclic subgroups of $G$ is denoted by $\mathcal{M}_{G}$. Note that $\left|\mathcal{M}_{G}\right|=1$ if and only if $G$ is cyclic. Denote by $\mathbb{Z}_{n}$ the cyclic group of order $n$. Let $\Gamma$ be a graph and $x \in V(\Gamma)$. The open neighborhood of $x$ in $\Gamma$ is

$$
N_{\Gamma}(x)=\{y \in V(\Gamma): d(y, x)=1\}
$$

and the closed neighborhood of $x$ in $\Gamma$ is

$$
N_{\Gamma}[x]=\{y \in V(\Gamma): d(y, x) \leq 1\} .
$$

If the situation is unambiguous, we denote $N_{\Gamma}(x)$ and $N_{\Gamma}[x]$ simply by $N(x)$ and $N[x]$, respectively.

Now we define a binary relation $x \approx y$ on $G$ by the rule that $N(x)=N(y)$ in $\mathcal{P}_{R}(G)$. It is readily seen that the relation is an equivalence relation over $G$. Let $\widehat{x}$ denote the equivalence $\approx$-class containing $x$. For $x \in G$, denote by $[x]$ the set of all generators of the cyclic subgroup $\langle x\rangle$, that is,

$$
[x]:=\{g \in G:\langle g\rangle=\langle x\rangle\} .
$$


Clearly, $\{[x]: x \in G\}$ is a partition of $G$.

Lemma 2.1. Let $x$ be an element of $G$. Then

(i) $[x] \subseteq \widehat{x}$. In particular, $x^{-1} \in \widehat{x}$.

(ii) $\widehat{x}$ is an independent set of $\mathcal{P}_{R}(G)$. In particular, $\widehat{e}=\{e\}$.

(iii) If there exists $y \in \widehat{x}$ such that $\langle y\rangle \notin \mathcal{M}_{G}$, then for any $M \in \mathcal{M}_{G}$ with $y \in M$, we have $\widehat{x} \subseteq M$.

Proof. (i) The proof is straightforward by the definition of a reduced power graph.

(ii) Suppose to the contrary that there exist distinct $a, b \in \widehat{x}$ such that $a$ and $b$ are adjacent in $\mathcal{P}_{R}(G)$. Without loss of generality, assume that $\langle a\rangle \subset\langle b\rangle$. Then $a \in N(b)=$ $N(a)$, a contradiction.

(iii) For any $x^{\prime} \in \widehat{x}$, we have that $N(y)=N\left(x^{\prime}\right)$. Let $M=\langle g\rangle$. Since $y \in M$ and $\langle y\rangle \notin \mathcal{M}_{G}$, we deduce that $g \in N(y)$, and so $g \in N\left(x^{\prime}\right)$. This means $x^{\prime} \in M$, and so $\widehat{x} \subseteq M$.

Recall now the following elementary result.

Theorem 2.2. [11, Theorem 5.4.10(ii)] Let p be a prime. Then a p-group having a unique subgroup of order $p$ is either cyclic or generalized quaternion.

For $x, y \in G$, define $x \equiv y$ if $N[x]=N[y]$ or $N(x)=N(y)$ in $\mathcal{P}_{R}(G)$. Hernando et al. [13] studied this relation and it follows from [13, Lemma 2.6] that $\equiv$ is an equivalence relation over $G$. Denote by $\bar{x}$ the $\equiv$-class containing the element $x \in G$. Let $\bar{G}=\{\bar{x}: x \in$ $G\}$.

For $n \geq 2$, Johnson [14] defined the generalized quaternion group $Q_{4 n}$ of order $4 n$ by the following presentation

$$
Q_{4 n}=\left\langle x, y: x^{n}=y^{2}, x^{2 n}=y^{4}=e, y^{-1} x y=x^{-1}\right\rangle .
$$

For $n=2$, the group $Q_{8}$ is the usual quaternion group of order 8, its another presentation is $Q_{8}=\{ \pm 1, \pm i, \pm j, \pm k\}$ with the operation $i^{2}=j^{2}=k^{2}=i j k=-1$. Remark that $Q_{4 n}$ has a unique involution $x^{n}$. Also,

$$
Q_{4 n}=\langle x\rangle \cup\left\{x^{i} y: 1 \leq i \leq 2 n\right\}, \quad o\left(x^{i} y\right)=4 \quad \text { for each } 1 \leq i \leq 2 n
$$

and

$$
\mathcal{M}_{Q_{4 n}}=\left\{\langle x\rangle,\langle x y\rangle, \ldots,\left\langle x^{n} y\right\rangle\right\}, \quad x^{n} \in \bigcap_{M \in \mathcal{M}_{Q_{4 n}}} M
$$


Lemma 2.3. Let $x$ and $y$ be two distinct elements of $G$. Then $N[x]=N[y]$ if and only if $G$ is isomorphic to either $\mathbb{Z}_{2^{m}}$ or $Q_{4 \cdot 2^{m}}$ where $m$ is a positive integer, and $\{x, y\}=\{e, a\}$ where $a$ is the unique involution of $G$.

Proof. If $G \cong \mathbb{Z}_{2^{m}}$, clearly, $N[e]=N[a]=G$ where $a$ is the unique involution of $G$, as desired. If $G \cong Q_{4 \cdot 2^{m}}$, it follows from 2.3 that $N[e]=N[a]=G$, where $a$ is the unique involution of $G$, as desired. Thus, the sufficiency follows.

We next prove the necessity. Suppose that $N[x]=N[y]$. Since $x \neq y$, we have $x \in N(y)=N\left(y^{-1}\right)$. It follows that $y^{-1} \in N[x]$, and so $y^{-1} \in N[y]$. Now the fact that $y^{-1}$ and $y$ are non-adjacent in $\mathcal{P}_{R}(G)$ would imply $y^{-1}=y$ and then $o(y) \leq 2$. Similarly, we can also deduce $o(x) \leq 2$. Note that $x$ is adjacent to $y$ in $\mathcal{P}_{R}(G)$. We have $\{x, y\}=\{e, a\}$ where $a$ is an involution. It follows that $N[a]=N[e]=G$. Now [2, Lemma 2.1] implies the desired result.

Corollary 2.4. $|\bar{e}| \leq 2$ with equality if and only if $G$ is isomorphic to either $\mathbb{Z}_{2^{m}}$ or $Q_{4 \cdot 2^{m}}$, where $m$ is a positive integer.

A group is called a $\mathcal{P}$-group [8] if every nontrivial element of the group has prime order. For example, the elementary abelian $p$-group $\mathbb{Z}_{p}^{n}$ is a $\mathcal{P}$-group where $p$ is a prime and $n \geq 1$, and the symmetric group $S_{3}$ on 3 letters is also a $\mathcal{P}$-group.

Lemma 2.5. Let $x \in G \backslash\{e\}$. Then

(i) If $|\bar{x}|=1$, then $o(x)=2$. The converse is not true.

(ii) $|\bar{G}|=1$ if and only if $G \cong \mathbb{Z}_{2}$, which in turn is true if and only if $\mathcal{P}_{R}(G)$ is complete.

(iii) $|\bar{G}|=2$ if and only if $G$ is isomorphic to $\mathbb{Z}_{4}, Q_{8}$, or a $\mathcal{P}$-group.

Proof. (i) By Lemma 2.1(i), we have $x^{-1} \in \widehat{x} \subseteq \bar{x}$. So $x^{-1}=x$, which implies $o(x)=2$. For the converse, considering $S_{3}$, we have $\overline{(12)}=S_{3} \backslash\{(1)\}$.

(ii) Clearly, $G \cong \mathbb{Z}_{2}$ if and only if $\mathcal{P}_{R}(G)$ is complete. Now suppose that $|\bar{G}|=1$. Then $x \in \bar{e}$. Since $x$ and $e$ are adjacent in $\mathcal{P}_{R}(G)$, we deduce $N[x]=N[e]$. It follows from Lemma 2.3 that $G \cong \mathbb{Z}_{2}$, as desired. Also, it is clear that $\left|\overline{\mathbb{Z}_{2}}\right|=1$.

(iii) Let $\mathbb{Z}_{4}=\langle g\rangle$ and let $H$ be a $\mathcal{P}$-group. Note that $\mathcal{P}_{R}(H)$ is a star. It is easy to check that $\overline{\mathbb{Z}_{4}}=\left\{\left\{e, g^{2}\right\},\left\{g, g^{3}\right\}\right\}, \overline{Q_{8}}=\left\{\{e, x\}, Q_{8} \backslash\{e, x\}\right\}$ where $x$ is the unique involution of $Q_{8}$, and $\bar{H}=\{\{e\}, H \backslash\{e\}\}$.

Now suppose that $|\bar{G}|=2$. Assume that $|\bar{e}|=1$. Then $\bar{x}=G \backslash\{e\}$. Suppose for a contradiction that there exists $y \in G$ such that $o(y)=p^{2}$ for some prime $p$. Then $o\left(y^{p}\right)=p$ and $y^{p} \in \bar{x}=\bar{y}$. Note that Lemma 2.3 implies $N[y] \neq N\left[y^{p}\right]$. It follows that 
$N(y)=N\left(y^{p}\right)$. As a result, we have $y^{-1} \in N\left(y^{p}\right)=N(y)$. However, $y^{-1}$ and $y$ are nonadjacent in $\mathcal{P}_{R}(G)$, a contradiction. Thus, in this case we deduce that $G$ is a $\mathcal{P}$-group, as desired.

Assume now that $|\bar{e}| \geq 2$. Corollary 2.4 implies that $|\bar{e}|=2$, and $G$ is isomorphic to either $\mathbb{Z}_{2^{m}}$ or $Q_{4 \cdot 2^{m}}$, where $m$ is a positive integer. Now Lemmas 2.1(ii) and 2.3 imply that $G \backslash \bar{e}$ is an independent set of $\mathcal{P}_{R}(G)$. Note that $\bar{e}=\{e, a\}$ where $a$ is the unique involution of $G$. Thus, $G$ can not have an element $z$ of order 8 , for otherwise $G \backslash \bar{e}$ contains the adjacent vertices $z$ and $z^{2}$, a contradiction. It follows that $G$ is isomorphic to either $\mathbb{Z}_{4}$ or $Q_{8}$, as desired.

Lemma 2.6. Let $|\bar{G}| \geq 3$. Then there exist distinct $\bar{a}, \bar{b} \in \bar{G}$ such that $|\bar{a}| \geq 2$ and $|\bar{b}| \geq 2$.

Proof. By Lemma 2.5(iii), we have that $G$ is not a $\mathcal{P}$-group. Hence we may assume that $G$ has an element $a$ of order $p^{2}$, where $p$ is a prime. If $p \geq 3$, then $|\bar{a}| \geq 2$ and $\left|\overline{a^{p}}\right| \geq 2$, and the desired result follows since $\bar{a} \neq \overline{a^{p}}$. In the following we may assume that $p=2$. Note that $G \nsubseteq \mathbb{Z}_{4}$.

Assume that there exists $c \in G \backslash\langle a\rangle$ such that $o(c) \neq 2^{m}$ for any positive integer $m$. Then $|\bar{c}| \geq 2$. We next prove $\bar{a} \neq \bar{c}$. Suppose for a contradiction that $\bar{a}=\bar{c}$. Then Lemma 2.3 implies $N(a)=N(c)$. Since $o(a)=4$, we have $\left\langle a^{2}\right\rangle \subset\langle a\rangle$, and so $\left\langle a^{2}\right\rangle \subset\langle c\rangle$. Thus, we may assume that $o(c)=2^{l} k$ for some positive integer $l$ and odd integer $k$ at least 3. Note that $o\left(c^{2^{l}}\right)=k$ is odd and $c^{2^{l}} \in N(c)=N(a)$. It follows that either $\left\langle c^{2^{l}}\right\rangle \subset\langle a\rangle$ or $\langle a\rangle \subset\left\langle c^{2^{l}}\right\rangle$, a contradiction as $o(a)=4$. Hence, in this case, $\bar{a}$ and $\bar{c}$ are the desired equivalence classes. As a consequence, in the following we assume that $G$ is a 2 -group.

Suppose that $G$ has a unique subgroup of order 2. By Theorem 2.2, we have that $G$ is either cyclic or generalized quaternion. Also, note that $G ¥ \mathbb{Z}_{4}$ or $Q_{8}$ by Lemma 2.5(iii). It follows that $G$ has an element $b$ of order 8 such that $a \in\langle b\rangle$. As a result, $|\bar{b}| \geq 2$ and $\bar{a} \neq \bar{b}$, as desired.

Now suppose that $G$ has at least two elements of order 2. Since the number of involutions of a finite group of even order is odd, we may assume that there exist distinct $u, v \in G \backslash\left\{a^{2}\right\}$ such that $o(u)=o(v)=2$. If $\langle u\rangle,\langle v\rangle \in \mathcal{M}_{G}$, then $v \in \bar{u}$ and so $|\bar{u}| \geq 2$, as desired. So we may assume that there exists $\langle b\rangle \in \mathcal{M}_{G}$ such that one of $u$ and $v$ belongs to $\langle b\rangle$. Since $o(b) \geq 4$, we have that $|\bar{b}| \geq 2$ and $\bar{a} \neq \bar{b}$.

For a positive integer $n$, let $D(n)$ denote the set of all divisors of $n$ and let $\sigma_{n}$ be the cardinality of $D(n)$, that is, $\sigma_{n}=|D(n)|$. Denote by $\phi$ the Euler's totient function. Recall that if $n$ is a positive integer and $n=p_{1}^{\lambda_{1}} p_{2}^{\lambda_{2}} \cdots p_{v}^{\lambda_{v}}$ is its canonical factorization (that is, $p_{1}, p_{2}, \ldots, p_{v}$ are distinct primes and $\lambda_{i} \geq 1$ for each $\left.1 \leq i \leq v\right)$, then $\sigma_{n}=\prod_{i=1}^{v}\left(\lambda_{i}+1\right)$.

The next lemma determines the number of all $\equiv$-classes of a cyclic group. 
Lemma 2.7. Let $n$ be a positive integer at least 2. Then

$$
\left|\overline{\mathbb{Z}_{n}}\right|= \begin{cases}3 & \text { if } n=p q \text { for two distinct primes } p \text { and } q, \\ m & \text { if } n=2^{m} \text { for some } m \geq 1 \\ \sigma_{n} & \text { otherwise. }\end{cases}
$$

Proof. For two distinct primes $p$ and $q$, it is easy to check that $\overline{\mathbb{Z}_{p q}}=\{\{e\}, A, B\}$, where $A$ and $B$ are the set of all elements of prime order and the set of all generators of $\mathbb{Z}_{p q}$, respectively. Now for $\mathbb{Z}_{2^{m}}$, let $A_{i}=\left\{x \in \mathbb{Z}_{2^{m}}: o(x)=2^{i}\right\}$ for each $0 \leq i \leq m$, then by Lemmas 2.3 and 2.1 (ii), we deduce that $\overline{\mathbb{Z}_{2^{m}}}=\left\{A_{0} \cup A_{1}, A_{2}, A_{3}, \ldots, A_{m}\right\}$, as desired.

In the following, suppose that $n$ is neither a power of 2 nor a product of two distinct primes. Let $x, y \in \mathbb{Z}_{n}$ with $x \equiv y$. Then Lemma 2.3 implies $N(x)=N(y)$. It follows from [2, Lemma 2.2] that either $\langle x\rangle=\langle y\rangle$, or $o(x)$ and $o(y)$ are distinct primes.

Suppose, for a contradiction, that $o(x)=p$ and $o(y)=q$, where $p, q$ are two distinct primes. Since $n \neq p q$, there exists $w \in \mathbb{Z}_{n}$ such that $o(w)=p^{2}$ or $p r$ (respectively $q^{2}$ or $q r$ ) where $r$ is a prime distinct from $p$ and $q$. It follows that $w \in N(x)$ (respectively $w \in N(y)$ ), and so $w \in N(y)=N(x)$ (respectively $w \in N(x)=N(y)$ ), a contradiction.

Thus, by Lemma 2.1(i), we conclude that $x \equiv y$ if and only if $\langle x\rangle=\langle y\rangle$. Let $D(n)=$ $\left\{d_{1}, d_{2}, \ldots, d_{\sigma_{n}}\right\}$. We deduce that $\overline{\mathbb{Z}_{n}}=\left\{\overline{x_{1}}, \overline{x_{2}}, \ldots, \overline{x_{\sigma_{n}}}\right\}$, where $\overline{x_{i}}=\left\{x \in \mathbb{Z}_{n}: o(x)=d_{i}\right\}$ for each $1 \leq i \leq \sigma_{n}$.

By the proof of Lemma 2.7, we have the following remark.

Remark 2.8. (i) Let $m$ be a positive integer. Then $\overline{\mathbb{Z}_{2^{m}}}=\left\{A_{0} \cup A_{1}, A_{2}, A_{3}, \ldots, A_{m}\right\}$, where $A_{i}=\left\{x \in \mathbb{Z}_{2^{m}}: o(x)=2^{i}\right\}$ for each $0 \leq i \leq m$.

(ii) Let $p, q$ be distinct primes. Then $\overline{\mathbb{Z}_{p q}}=\{\{e\}, A, B\}$, where $A=\left\{x \in \mathbb{Z}_{p q}: o(x)=\right.$ $p$ or $q\}$ and $B=\left\{x \in \mathbb{Z}_{p q}: o(x)=p q\right\}$.

(iii) Suppose that $n$ is neither a power of 2 nor a product of two distinct primes. Let $D(n)=\left\{d_{1}, d_{2}, \ldots, d_{\sigma_{n}}\right\}$ and $S_{i}=\left\{x \in \mathbb{Z}_{n}: o(x)=d_{i}\right\}$ for each $1 \leq i \leq \sigma_{n}$. Then $\overline{\mathbb{Z}_{n}}=\left\{S_{1}, S_{2}, \ldots, S_{\sigma_{n}}\right\}$.

Let $G$ be a group. Define

$$
\mathcal{L}_{G}:=\{g \in G: o(g)=2 \text { and } \bar{g}=\{g\}\} .
$$

For example, $\mathcal{L}_{Q_{12}}=\left\{x^{3}\right\}$ by (2.4), and if $H=\langle h\rangle \cong \mathbb{Z}_{12}$, then $\mathcal{L}_{H}=\left\{h^{6}\right\}$ by Remark 2.8(iii). Also, for $a, b \in G$, define

$$
R\{a, b\}:=\{x \in G: d(a, x) \neq d(b, x)\}
$$

which is the set of vertices resolving $a$ and $b$ in $\mathcal{P}_{R}(G)$. From Lemma 2.5(ii), it follows that $\mathcal{P}_{R}(G)$ is complete if and only if $G \cong \mathbb{Z}_{2}$. If $G \nsubseteq \mathbb{Z}_{2}$, then in $\mathcal{P}_{R}(G)$, for distinct 
$x, y \in G$ with $d(x, y) \neq 1$, we have $d(x, y)=2$, because $e$ is adjacent to every vertex in $G \backslash\{e\}$. Notice that in a group $G$, if $x \in G$ and $|\bar{x}| \geq 2$, then every pair of distinct vertices of $\bar{x}$ is resolved by some element of $\bar{x} \backslash\{x\}$. According to this, it suffices to determine the resolvability between $\equiv$-classes of $G$ if the resolving set consists all $\bar{x} \backslash\{x\}$ with $|\bar{x}| \geq 2$.

Proposition 2.9. Let $p$ be an odd prime and $\left\{x_{1}, x_{2}, \ldots, x_{6}\right\}$ be a system of representatives for the $\equiv$-classes of $\mathbb{Z}_{2 p^{2}}$. Then $S=\left(\mathbb{Z}_{2 p^{2}} \backslash\left\{x_{1}, x_{2}, \ldots, x_{6}\right\}\right) \cup \mathcal{L}_{\mathbb{Z}_{2 p^{2}}}$ is a resolving set of $\mathcal{P}_{R}\left(\mathbb{Z}_{2 p^{2}}\right)$.

Proof. By Remark 2.8(iii), we may assume that $x_{1}=e, \overline{x_{2}}=\left\{g \in \mathbb{Z}_{2 p^{2}}: o(g)=2\right\}=\left\{x_{2}\right\}$, $\overline{x_{3}}=\left\{g \in \mathbb{Z}_{2 p^{2}}: o(g)=p\right\}, \overline{x_{4}}=\left\{g \in \mathbb{Z}_{2 p^{2}}: o(g)=2 p\right\}, \overline{x_{5}}=\left\{g \in \mathbb{Z}_{2 p^{2}}: o(g)=p^{2}\right\}$ and $\overline{x_{6}}=\left\{g \in \mathbb{Z}_{2 p^{2}}: o(g)=2 p^{2}\right\}$. So, $\mathcal{L}_{\mathbb{Z}_{2 p^{2}}}=\left\{x_{2}\right\}$. Now it is easy to check that $x_{3}^{-1} \in S \cap R\left\{x_{1}, x_{3}\right\}, x_{4}^{-1} \in S \cap R\left\{x_{1}, x_{4}\right\} \cap R\left\{x_{3}, x_{5}\right\} \cap R\left\{x_{5}, x_{6}\right\}, x_{5}^{-1} \in S \cap R\left\{x_{1}, x_{5}\right\} \cap$ $R\left\{x_{3}, x_{4}\right\} \cap R\left\{x_{4}, x_{6}\right\}, x_{6}^{-1} \in S \cap R\left\{x_{1}, x_{6}\right\} \cap R\left\{x_{3}, x_{6}\right\}$, and $x_{2} \in S \cap R\left\{x_{4}, x_{5}\right\}$, as desired.

Proposition 2.10. Let $n$ be a positive integer at least 3 and $\left\{x_{1}, x_{2}, \ldots, x_{t}\right\}$ be a system of representatives for the $\equiv$-classes of $\mathbb{Z}_{n}$. If $n \neq 2 p^{2}$ for some odd prime $p$, then $S=$ $\mathbb{Z}_{n} \backslash\left\{x_{1}, x_{2}, \ldots, x_{t}\right\}$ is a resolving set of $\mathcal{P}_{R}\left(\mathbb{Z}_{n}\right)$.

Proof. We divide our proof into three cases.

Case 1: $n=p q$ for two distinct primes $p, q$.

By Remark 2.8(ii), we may assume that $\left\{e, x_{2}, x_{3}\right\}$ is a system of representatives for the $\equiv$-classes of $\overline{\mathbb{Z}_{n}}$, where $x_{2}$ is an element of order $p$ or $q$, and $x_{3}$ is an element of order $p q$. Let $x \in \mathbb{Z}_{n}$ be an element of prime order such that $o(x) \neq o\left(x_{2}\right)$. Clearly, $d\left(x, x_{2}\right)=2$ and $d\left(x, x_{3}\right)=1$, and so $x \in R\left\{e, x_{2}\right\} \cap S \cap R\left\{x_{2}, x_{3}\right\}$. Also, from $o\left(x_{3}\right) \geq 6$ and $d\left(x_{3}^{-1}, x_{3}\right)=2$, it follows that $x_{3}^{-1} \in R\left\{e, x_{3}\right\} \cap S$. This means that $\mathbb{Z}_{n} \backslash\left\{e, x_{2}, x_{3}\right\}$ is a resolving set of $\mathcal{P}_{R}\left(\mathbb{Z}_{n}\right)$, as desired.

Case 2: $n=2^{m}$ for some positive integer $m \geq 2$.

Let $A_{i}=\left\{x \in \mathbb{Z}_{2^{m}}: o(x)=2^{i}\right\}$ for each $1 \leq i \leq m$. In view of Remark 2.8(i), we may assume that $\left\{x_{1}, x_{2}, \ldots, x_{m}\right\}$ is a system of representatives for the $\equiv$-classes of $\overline{\mathbb{Z}_{n}}$, where $x_{i} \in A_{i}$ for each $1 \leq i \leq m$. Now let $a$ and $b$ be two distinct elements of $\left\{x_{1}, x_{2}, \ldots, x_{m}\right\}$, it suffices to prove that there exists $s \in S$ such that $s \in R\{a, b\}$. Without loss of generality, we may assume that $o(a)<o(b)$. Then $d(a, b)=1$ and $o(b) \geq 4$. It follows that $b^{-1} \in S$ and $d\left(b, b^{-1}\right)=2$, and so $b^{-1} \in R\{a, b\}$, as desired.

Case 3: $n$ is neither a power of 2 nor a product of two distinct primes.

Let $D(n)=\left\{d_{1}, d_{2}, \ldots, d_{\sigma_{n}}\right\}$ and $S_{i}=\left\{x \in \mathbb{Z}_{n}: o(x)=d_{i}\right\}$ for each $1 \leq i \leq \sigma_{n}$, where $d_{1}=1$. Then Remark 2.8 (iii) implies that we may assume that $\left\{x_{1}, x_{2}, \ldots, x_{\sigma_{n}}\right\}$ is a system of representatives for the $\equiv$-classes of $\overline{\mathbb{Z}_{n}}$, where $x_{i} \in S_{i}$ for each $1 \leq i \leq \sigma_{n}$. Since $\mathbb{Z}_{n}$ is not a 2 -group, we may choose $y \in S$ such that $o(y)$ is an odd prime by 
Lemma 2.1(i). Let $x$ be an element of $\left\{x_{2}, \ldots, x_{\sigma_{n}}\right\}$. Note that $x_{1}=e$. If $o(x)=2$, then $y \in S \cap R\left\{x_{1}, x\right\}$. If $o(x) \geq 3$, then $x^{-1} \in S \cap R\left\{x_{1}, x\right\}$. Thus, let $a$ and $b$ be two distinct elements of $\left\{x_{2}, \ldots, x_{\sigma_{n}}\right\}$, it suffices to prove that there exists $s \in S$ such that $s \in R\{a, b\}$.

If $a$ and $b$ are adjacent in $\mathcal{P}_{R}\left(\mathbb{Z}_{n}\right)$, then $|\bar{a}|$ or $|\bar{b}| \geq 2$, and so $a^{-1}$ or $b^{-1} \in R\{a, b\} \cap S$, as desired. Thus, in the following we may assume that $a$ and $b$ are non-adjacent. Note that $n \neq 2^{m}, p q, 2 p^{2}$ where $p, q$ are distinct primes and $m$ is a positive integer. We conclude that there exists an integer $l>2$ such that $l \mid n$ and one of the following holds:

(i) $l \mid o(a), l \neq o(a)$ and $l \nmid o(b)$;

(iii) $o(a) \mid l, l \neq o(a)$ and $o(b) \nmid l$;

(ii) $l \mid o(b), l \neq o(b)$ and $l \nmid o(a)$;

(iv) $o(b) \mid l, l \neq o(b)$ and $o(a) \nmid l$.

Now let $z \in \mathbb{Z}_{n}$ with $o(z)=l$. Note that $a$ and $b$ are non-adjacent. It follows that one of $z$ and $z^{-1}$ must belong to $S \cap R\{a, b\}$, as desired.

Proposition 2.11. Let $Q_{4 n}$ be the generalized quaternion group as presented in (2.1) and $\left\{x_{1}, x_{2}, \ldots, x_{t}\right\}$ be a system of representatives for the $\equiv$-classes of $Q_{4 n}$. If $n \neq p^{2}$ for some odd prime $p$, then $S=Q_{4 n} \backslash\left\{x_{1}, x_{2}, \ldots, x_{t}\right\}$ is a resolving set of $\mathcal{P}_{R}\left(Q_{4 n}\right)$.

Proof. Suppose that $n=2$. By Lemma 2.5 (iii), $\overline{Q_{8}}=\left\{\left\{e, x^{2}\right\}, Q_{8} \backslash\left\{e, x^{2}\right\}\right\}$. Let $\left\{x_{1}, x_{2}\right\}$ be a system of representatives for the $\equiv$-classes of $Q_{8}$, where $x_{1}=e$ or $x^{2}, x_{2} \in Q_{8} \backslash\left\{e, x^{2}\right\}$. Then $x_{2}^{-1} \in S \cap R\left\{x_{1}, x_{2}\right\}$, as desired.

Suppose that $n=3$. It is easy to check that

$$
\overline{Q_{12}}=\left\{\{e\},\left\{x^{3}\right\},\left\{x^{2}, x^{4}\right\},\left\{x, x^{5}\right\}, Q_{12} \backslash\langle x\rangle\right\} .
$$

Let $\left\{x_{1}, x_{2}, x_{3}, x_{4}, x_{5}\right\}$ be a system of representatives for the $\equiv$-classes of $Q_{8}$, where $x_{1}=e$, $x_{2}=x^{3}, x_{3} \in\left\{x^{2}, x^{4}\right\}, x_{4} \in\left\{x, x^{5}\right\}$ and $x_{5} \in Q_{12} \backslash\langle x\rangle$. Then $x_{5}^{-1} \in S \cap R\left\{x_{1}, x_{5}\right\} \cap$ $R\left\{x_{2}, x_{5}\right\} \cap R\left\{x_{2}, x_{3}\right\}, x_{4}^{-1} \in S \cap R\left\{x_{3}, x_{5}\right\} \cap R\left\{x_{1}, x_{4}\right\} \cap R\left\{x_{2}, x_{4}\right\} \cap R\left\{x_{3}, x_{4}\right\}$, and $x_{3}^{-1} \in S \cap R\left\{x_{4}, x_{5}\right\} \cap R\left\{x_{1}, x_{2}\right\} \cap R\left\{x_{1}, x_{3}\right\}$, as desired.

Now suppose that $n \geq 4$. Note that $\langle x\rangle \cong \mathbb{Z}_{2 n}$. By Remark 2.8, (2.2) and (2.3), we deduce that

$$
\overline{Q_{4 n}}=\overline{\langle x\rangle} \cup\left\{Q_{4 n} \backslash\langle x\rangle\right\} .
$$

So we may assume that $\left\{x_{1}, x_{2}, \ldots, x_{t-1}, g\right\}$ is a system of representatives for the $\equiv$ classes of $Q_{4 n}$, where $g=x_{t} \in\left\{Q_{4 n} \backslash\langle x\rangle\right\}$ and $\left\{x_{1}, x_{2}, \ldots, x_{t-1}\right\}$ is a system of representatives for the $\equiv$-classes of $\langle x\rangle$. Note that $g$ has order 4 and $N(g)=\left\{e, x^{n}\right\}$. Let $h \in\left\{x_{1}, x_{2}, \ldots, x_{t-1}\right\}$. If $h \notin[x]$, then $x, x^{-1} \in R\{g, h\}$, which implies that either $x$ or $x^{-1}$ belongs to $S \cap R\{g, h\}$ since $x^{-1} \in \bar{x}$. Suppose that $h \in[x]$. Since $n \neq 2$, there exists an element $f \in\langle x\rangle \backslash[x]$ such that $o(f) \geq 3$. It follows that one of $f$ and $f^{-1}$ must belong to $S \cap R\{g, h\}$. Now in order to complete the proof, it suffices to show that there exists an element in $S$ such that it resolves every pair of distinct vertices of $\left\{x_{1}, x_{2}, \ldots, x_{t-1}\right\}$. The desired result follows from Proposition 2.10. 


\section{The metric dimension of a reduced power graph}

In this section, we give tight upper and lower bounds for $\operatorname{dim}\left(\mathcal{P}_{R}(G)\right.$ ) (see Theorem 3.2 ). As applications, we compute the exact value of $\operatorname{dim}\left(\mathcal{P}_{R}(G)\right)$ if $G$ is a $\mathcal{P}$-group, a cyclic group, a dihedral group and a generalized quaternion group.

Proposition 3.1. Let $G$ be a group with $|\bar{G}| \geq 2$, and let $\left\{x_{1}, x_{2}, \ldots, x_{r}\right\}$ be a system of representatives for the $\equiv$-classes of $G$. Then

$$
S=\left(G \backslash\left\{x_{1}, x_{2}, \ldots, x_{r}\right\}\right) \cup \mathcal{L}_{G}
$$

is a resolving set of $\mathcal{P}_{R}(G)$.

Proof. By Propositions 2.10 and 2.11, it is easy to see that the desired result follows for $\mathbb{Z}_{2^{m}}$ and $Q_{4 \cdot k}$ where $m \geq 2$ and $k$ is a power of 2 . In the following, we assume that $G$ is neither $\mathbb{Z}_{2^{m}}$ nor $Q_{4 \cdot k}$. Since $|\bar{G}| \geq 2$, we have $G \neq \mathbb{Z}_{2}$ by Lemma 2.5(ii). Also, it follows from Lemma 2.3 that for two distinct $x, y \in G, x \equiv y$ if and only if $x \approx y$ in $\mathcal{P}_{R}(G)$. So Lemma 2.1(ii) implies that every $\equiv$-class is an independent set of $\mathcal{P}_{R}(G)$ and $\bar{e}=\{e\}$. Now, let $a$ and $b$ be two distinct elements of $\left\{x_{1}, x_{2}, \ldots, x_{r}\right\}$ with $a, b \notin \mathcal{L}_{G}$, it suffices to prove that there exists $s \in S$ such that $s \in R\{a, b\}$. Observe that $\bar{a} \neq \bar{b}$.

Suppose that one of $a$ and $b$ is $e$, without loss of generality, say $a=e$. Since $b \notin \mathcal{L}_{G}$, we have $|\bar{b}| \geq 2$. Taking $b^{\prime} \in \bar{b} \backslash\{b\}$, we have that $d\left(b, b^{\prime}\right)=2$ since $\bar{b}$ is an independent set of $\mathcal{P}_{R}(G)$. It follows that $b^{\prime} \in S \cap R\{a, b\}$.

Thus, in the following we always assume that $a \neq e$ and $b \neq e$. We divide our proof into two cases.

Case 1: For any $M \in \mathcal{M}_{G},\{a, b\} \nsubseteq M$.

Let $M_{1}, M_{2} \in \mathcal{M}_{G}$ with $a \in M_{1}$ and $b \in M_{2}$. Clearly, $M_{1} \neq M_{2}$. Suppose that $\langle a\rangle \neq M_{1}$. Let $M_{1}=\left\langle m_{1}\right\rangle$. Then $d\left(m_{1}, a\right)=1$. If $d\left(m_{1}, b\right)=1$, then $b \in M_{1}$, and so $a, b \in M_{1}$, a contradiction. It follows that $d\left(m_{1}, b\right)=2$ and so $m_{1}, m_{1}^{-1} \in R\{a, b\}$. Also, note that $o\left(m_{1}\right) \geq 4$, we have that $\left|\overline{m_{1}}\right| \geq 2$ by Lemma $2.1(\mathrm{i})$. As a result, one of $m_{1}$ and $m_{1}^{-1}$ must belong to $S$, as desired. Similarly, if $\langle b\rangle \neq M_{2}$, we also can obtain the desired result. Hence, in the following, we assume that $\langle a\rangle=M_{1}$ and $\langle b\rangle=M_{2}$. Note that $N(a) \neq N(b)$. Suppose that there exists $x \in N(a)$ such that $x \notin N(b)$. Then $x \in R\{a, b\}$. Clearly, $x \neq e$. If $|\bar{x}|=1$, then $x \in \mathcal{L}_{G}$, and so $x \in S$, as desired. Thus, we may assume that $|\bar{x}| \geq 2$. Taking $y \in \bar{x} \backslash\{x\}$, we have $b \notin N(y)$, otherwise $b \in N(y)=N(x)$, contrary to $x \notin N(b)$. Since $y \in N(a)$, we have $y \in R\{a, b\}$. Now by the definition of $S$, we deduce that $x \in S$ or $y \in S$, as desired.

Case 2: There exists $M \in \mathcal{M}_{G}$ such that $\{a, b\} \subseteq M$.

If $M=\langle a\rangle$, clearly $o(a) \geq 4$, and so $a^{-1} \in S \cap R\{a, b\}$ as $M \neq\langle b\rangle$. Similarly, if $M=\langle b\rangle$, the desired result also follows. Thus, we may assume that $\langle a\rangle \subset M$ and $\langle b\rangle \subset M$. Now Lemma 2.1(iii) implies that $\bar{a}, \bar{b} \subseteq M$. 
Suppose that $|M|=p q$ for two distinct primes $p, q$. Then $\{o(a), o(b)\}=\{p, q\}$. Since $N(a) \neq N(b)$, there exists $\langle g\rangle \in \mathcal{M}_{G} \backslash\{M\}$ such that $a \in\langle g\rangle$ or $b \in\langle g\rangle$. If $a \in\langle g\rangle$ and $b \notin\langle g\rangle$, then it is easy to see that one of $g$ and $g^{-1}$ must belong to $S \cap R\{a, b\}$, as desired. Similarly, if $a \notin\langle g\rangle$ and $b \in\langle g\rangle$, we also can obtain the desired result. So, we may assume that $a \in\langle g\rangle$ and $b \in\langle g\rangle$, which implies $M \subseteq\langle g\rangle$ and hence $M=\langle g\rangle$, a contradiction. Now we may assume that $|M|$ is not a product of two distinct primes. Note that if $|M|$ is a power of 2 , then the involution of $M$ belongs to $\mathcal{L}_{G}$. It follows from Remark 2.8(i) and (iii) that $\bar{a}$ and $\bar{b}$ are two distinct $\equiv$-class of $M$. Now Propositions 2.9 and 2.10 imply the desired result.

Theorem 3.2. Let $G$ be a group of order $n$. Then

$$
n-|\bar{G}| \leq \operatorname{dim}\left(\mathcal{P}_{R}(G)\right) \leq n-|\bar{G}|+\left|\mathcal{L}_{G}\right|
$$

Proof. If $|\bar{G}|=1$, then $\mathcal{P}_{R}(G)$ is complete by Lemma 2.5(ii), and it follows from 7 , Theorem 3] that $\operatorname{dim}\left(\mathcal{P}_{R}(G)\right)=n-1$, as desired. Now suppose that $|\bar{G}| \geq 2$. If $G$ is a $\mathcal{P}$-group, then $|\bar{G}|=2$ and $\mathcal{P}_{R}(G)$ is a star, and so $\operatorname{dim}\left(\mathcal{P}_{R}(G)\right)=n-2$ by 7 , Theorem 4], as desired. Thus, we may assume that $G$ is not a $\mathcal{P}$-group. Combining Lemmas 2.5(iii) and 2.6, we have that $G$ has two distinct $\equiv$-classes having size at least 2 , and so $n-|\bar{G}| \geq 2$. Let $S$ be a resolving set of $\mathcal{P}_{R}(G)$ with size $\operatorname{dim}\left(\mathcal{P}_{R}(G)\right)$. If there exist distinct elements $x, y \in G$ such that $\bar{x}=\bar{y}$ and $x, y \notin S$, then no element of $S$ resolves $x$ and $y$, a contradiction. Therefore, it follows that for each $\bar{g} \in \bar{G}$, we have that $\bar{g}$ has at most one element that does not belong to $S$. As a consequence, we deduce $|S| \geq n-|\bar{G}|$. Namely, $\operatorname{dim}\left(\mathcal{P}_{R}(G)\right) \geq n-|\bar{G}|$, as desired. Also, Proposition 3.1 implies that $\operatorname{dim}\left(\mathcal{P}_{R}(G)\right) \leq n-|\bar{G}|+\left|\mathcal{L}_{G}\right|$. Now the proof is complete.

The following result is immediate by Theorem 3.2 .

Corollary 3.3. Let $G$ be a group of order $n$. If $n$ is odd, then $\operatorname{dim}\left(\mathcal{P}_{R}(G)\right)=n-|\bar{G}|$.

In the following, as applications of Theorem 3.2, we give some examples to illustrate that the bounds of 3.1 are tight.

Example 3.4. Let $G$ be a $\mathcal{P}$-group of order $n$. Then $\operatorname{dim}\left(\mathcal{P}_{R}(G)\right)=n-|\bar{G}|=n-2$.

Example 3.5. Let $n$ be a positive integer at least 2. Then

$$
\operatorname{dim}\left(\mathcal{P}_{R}\left(\mathbb{Z}_{n}\right)\right)= \begin{cases}n-3 & \text { if } n=p q \text { for two distinct primes } p \text { and } q \\ n-m & \text { if } n=2^{m} \text { for some } m \geq 1 \\ n-5 & \text { if } n=2 p^{2} \text { for some odd prime } p \\ n-\sigma_{n} & \text { otherwise. }\end{cases}
$$


Proof. Suppose that $n=2 p^{2}$ for some odd prime $p$. Then $\left|\overline{\mathbb{Z}_{n}}\right|=6$ and $\left|\mathcal{L}_{\mathbb{Z}_{n}}\right|=1$ by Proposition 2.9. Let $S$ be a resolving set of $\mathcal{P}_{R}\left(\mathbb{Z}_{n}\right)$ with size $\operatorname{dim}\left(\mathcal{P}_{R}\left(\mathbb{Z}_{n}\right)\right)$. Assume, by contradiction that $|S|=n-6$. Since it is impossible that there exist distinct elements $x, y \in G$ such that $\bar{x}=\bar{y}$ and $x, y \notin S$, we deduce that $\mathcal{L}_{\mathbb{Z}_{n}} \nsubseteq S$ and there exist distinct $a, b \notin S$ such that $a \in\left\{g \in \mathbb{Z}_{n}: o(g)=p^{2}\right\}$ and $b \in\left\{g \in \mathbb{Z}_{n}: o(g)=2 p\right\}$. This contradicts the fact $R\{a, b\}=\{a, b\} \cup \mathcal{L}_{\mathbb{Z}_{n}}$. We conclude that $\operatorname{dim}\left(\mathcal{P}_{R}\left(\mathbb{Z}_{n}\right)\right) \geq n-5$. Now Theorem 3.2 implies that $\operatorname{dim}\left(\mathcal{P}_{R}\left(\mathbb{Z}_{n}\right)\right)=n-5$.

Clearly, $\operatorname{dim}\left(\mathcal{P}_{R}\left(\mathbb{Z}_{2}\right)\right)=1$. In the following, suppose that $n \geq 3$ and $n \neq 2 p^{2}$ for some odd prime $p$. By Proposition 2.10 , we have $\operatorname{dim}\left(\mathcal{P}_{R}\left(\mathbb{Z}_{n}\right)\right) \leq n-\overline{\mathbb{Z}_{n}}$. Now Theorem 3.2 implies $\operatorname{dim}\left(\mathcal{P}_{R}\left(\mathbb{Z}_{n}\right)\right)=n-\overline{\mathbb{Z}_{n}}$. Thus, the desired result follows from Lemma 2.7 .

For $n \geq 3$, denote by $D_{2 n}$ the dihedral group of order $2 n$. Recall that a presentation of $D_{2 n}$ is given by

$$
D_{2 n}=\left\langle a, b: a^{n}=b^{2}=e, b a b=a^{-1}\right\rangle .
$$

Remark that $D_{2 n}=\langle a\rangle \cup\left\{b, a b, a^{2} b, \ldots, a^{n-1} b\right\}$, where $o\left(a^{i} b\right)=2$ and $N\left(a^{i} b\right)=\{e\}$ in $\mathcal{P}_{R}\left(D_{2 n}\right)$ for each $1 \leq i \leq n$. Thus, it is easy to see that $\overline{D_{2 n}}=\overline{\langle a\rangle} \cup \bar{b}$, where $\bar{b}=\left\{D_{2 n} \backslash\langle a\rangle\right\}$.

Remark 3.6. Observe that $\mathcal{P}_{R}\left(D_{2 n}\right)$ is a union of $\mathcal{P}_{R}\left(\mathbb{Z}_{n}\right)=\mathcal{P}_{R}(\langle a\rangle)$ and a star $K_{1, n}$ (a complete bipartite graph with partite sets $\{e\}$ and $\left.\left\{a^{i} b: 0 \leq i \leq n-1\right\}\right)$ with the common vertex $e$ (see [25, Fig. 2]). The union of resolving sets of two graphs still resolves vertices between two graphs. When $n$ is not a prime, every $\equiv$-class of $D_{2 n}$ entirely lies either $\mathcal{P}_{R}(\langle a\rangle)$ or the star. Thus, the metric dimension of $\mathcal{P}_{R}\left(D_{2 n}\right)$ is the sum of the metric dimensions of the two graphs. Since $\operatorname{dim}\left(K_{1, n}\right)=n-1$, it follows that $\operatorname{dim}\left(\mathcal{P}_{R}\left(D_{2 n}\right)\right)=$ $\operatorname{dim}\left(\mathcal{P}_{R}\left(\mathbb{Z}_{n}\right)\right)+(n-1)$. Notice that $\mathcal{P}_{R}\left(Q_{4 n}\right)$ is a union of $\mathcal{P}_{R}\left(\mathbb{Z}_{2 n}\right)$ and two copies of star $K_{1,2 n}$ with common vertices $e$ and an involution (see [25, Fig. 3]). Similarly, if $n \neq 2$, we can deduce that $\operatorname{dim}\left(\mathcal{P}_{R}\left(Q_{4 n}\right)\right)=\operatorname{dim}\left(\mathcal{P}_{R}\left(\mathbb{Z}_{2 n}\right)\right)+(2 n-1)$.

The following example follows from Examples 3.4 and 3.5 , and Remark 3.6 .

Example 3.7. Let $D_{2 n}$ be the dihedral group as presented in $(3.2)$. Then

$$
\operatorname{dim}\left(\mathcal{P}_{R}\left(D_{2 n}\right)\right)= \begin{cases}5 & \text { if } n=4, \\ 2 n-2 & \text { if } n \text { is an odd prime, } \\ 2 n-4 & \text { if } n=p q \text { for two distinct primes } p \text { and } q, \\ 2 n-6 & \text { if } n=2 p^{2} \text { for some odd prime } p, \\ 2 n-\sigma_{n}-1 & \text { otherwise. }\end{cases}
$$

It is clear that $\operatorname{dim}\left(\mathcal{P}_{R}\left(Q_{8}\right)\right)=6$ by Lemma 2.5 (iii), Proposition 2.11 and Theorem 3.2 . Now the following example follows from Example 3.5 and Remark 3.6. 
Example 3.8. Let $Q_{4 n}$ be the generalized quaternion group as presented in (2.1). Then

$$
\operatorname{dim}\left(\mathcal{P}_{R}\left(Q_{4 n}\right)\right)= \begin{cases}6 & \text { if } n=2, \\ 4 n-m-2 & \text { if } n=2^{m} \text { for } m \geq 2 \\ 4 n-6 & \text { if } n=p^{2} \text { for some odd prime } p \\ 4 n-\sigma_{2 n}-1 & \text { otherwise. }\end{cases}
$$

We conclude the paper by the following remark to illustrate Theorem 3.2

Remark 3.9. Let $p$ be an odd prime. Then every of $\operatorname{dim}\left(\mathcal{P}_{R}\left(\mathbb{Z}_{2 p^{2}}\right)\right)$, $\operatorname{dim}\left(\mathcal{P}_{R}\left(D_{2 \cdot 2 p^{2}}\right)\right)$ and $\operatorname{dim}\left(\mathcal{P}_{R}\left(Q_{4 p^{2}}\right)\right)$ can attain the upper bound of 3.1 . We remark that the metric dimension of a reduced power graph can lie in between the upper bound and lower bound. Let $G=\mathbb{Z}_{4} \times \mathbb{Z}_{4}$. The graph $\mathcal{P}_{R}(G)$ is displayed in Figure 3.1 .

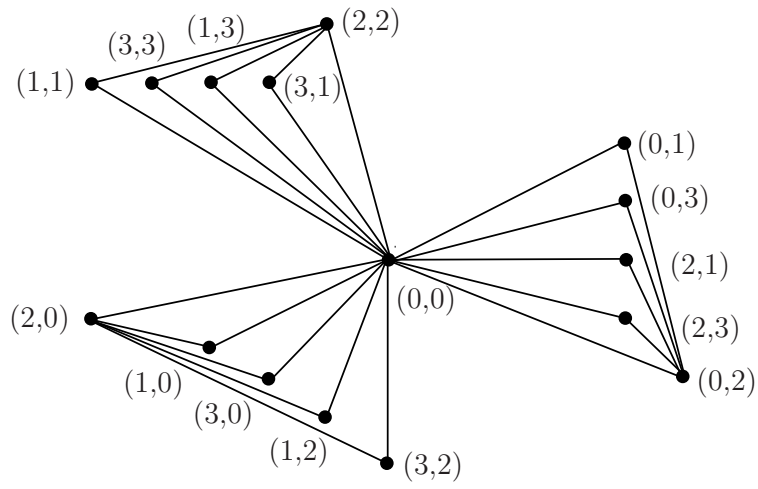

Figure 3.1: $\mathcal{P}_{R}\left(\mathbb{Z}_{4} \times \mathbb{Z}_{4}\right)$.

Then it is easy to see that $\bar{G}=\{\{(0,0)\},\{(0,2)\},\{(2,2)\},\{(2,0)\}, \overline{(0,1)}, \overline{(1,0)}, \overline{(1,1)}\}$, where $\overline{(0,1)}=\{(0,1),(0,3),(2,1),(2,3)\}, \overline{(1,0)}=\{(1,0),(3,0),(1,2),(3,2)\}$, and $\overline{(1,1)}=$ $\{(1,1),(3,3),(1,3),(3,1)\}$. Thus, $|\bar{G}|=7$ and $\left|\mathcal{L}_{G}\right|=3$. Also, we have $\operatorname{dim}\left(\mathcal{P}_{R}(G)\right) \geq 9$ by Theorem 3.2. Now let $S$ be a resolving set of $\mathcal{P}_{R}(G)$. Without loss of generality, we may assume that $\overline{(1,0)} \backslash\{(1,0)\} \subseteq S, \overline{(0,1)} \backslash\{(0,1)\} \subseteq S$, and $\overline{(1,1)} \backslash\{(1,1)\} \subseteq S$. If $S=(\overline{(1,0)} \backslash\{(1,0)\}) \cup \overline{((0,1)} \backslash\{(0,1)\}) \cup(\overline{(1,1)} \backslash\{(1,1)\})$, then no element of $S$ resolves $(1,0)$ and $(0,1)$, a contradiction. It follows that $|S|>9$. On the other hand, it is easy to see that $(\overline{(1,0)} \backslash\{(1,0)\}) \cup(\overline{(0,1)} \backslash\{(0,1)\}) \cup(\overline{(1,1)} \backslash\{(1,1)\}) \cup\{(0,2),(2,0)\}$ is a resolving set of $\mathcal{P}_{R}(G)$. Thus, we have that

$$
|G|-|\bar{G}|<\operatorname{dim}\left(\mathcal{P}_{R}(G)\right)<|G|-|\bar{G}|+\left|\mathcal{L}_{G}\right| \text {. }
$$

In fact, one can prove $\operatorname{dim}\left(\mathcal{P}_{R}(G)\right)=11$. 


\section{Acknowledgments}

We are grateful to the referees for many detailed comments and thoughtful suggestions that have helped improve this paper substantially.

This research was supported by the National Natural Science Foundation of China (Grant No. 11801441), the Natural Science Basic Research Program of Shaanxi (Program Nos. 2020JQ-761 and 2021JM-399), and the Young Talent fund of University Association for Science and Technology in Shaanxi, China (Grant No. 20190507).

\section{References}

[1] J. Abawajy, A. Kelarev and M. Chowdhury, Power graphs: A survey, Electron. J. Graph Theory Appl. (EJGTA) 1 (2013), no. 2, 125-147.

[2] T. Anitha and R. Rajkumar, On the power graph and the reduced power graph of a finite group, Comm. Algebra 47 (2019), no. 8, 3329-3339.

[3] Characterization of groups with planar, toroidal or projective planar (proper) reduced power graphs, J. Algebra Appl. 19 (2020), no. 5, 2050099, 16 pp.

[4] D. Bubboloni, M. A. Iranmanesh and S. M. Shaker, On some graphs associated with the finite alternating groups, Comm. Algebra 45 (2017), no. 12, 5355-5373.

[5] P. J. Cameron, H. Guerra and Š. Jurina, The power graph of a torsion-free group, J. Algebraic Combin. 49 (2019), no. 1, 83-98.

[6] I. Chakrabarty, S. Ghosh and M. K. Sen, Undirected power graphs of semigroups, Semigroup Forum 78 (2009), no. 3, 410-426.

[7] G. Chartrand, L. Eroh, M. A. Johnson and O. R. Oellermann, Resolvability in graphs and the metric dimension of a graph, Discrete Appl. Math. 105 (2000), no. 1-3, 99-113.

[8] M. Deaconescu, Classification of finite groups with all elements of prime order, Proc. Amer. Math. Soc. 106 (1989), no. 3, 625-629.

[9] M. Feng, X. Ma and K. Wang, The structure and metric dimension of the power graph of a finite group, European J. Combin. 43 (2015), 82-97.

[10] M. R. Garey and D. S. Johnson, Computers and Intractability: A guide to the theory of NP-completeness, A Series of Books in the Mathematical Sciences, W. H. Freeman and Co., New York, 1979. 
[11] D. Gorenstein, Finite Groups, Second edition, Chelsea Publishing Co., New York, 1980.

[12] F. Harary and R. A. Melter, On the metric dimension of a graph, Ars Combin. 2 (1976), 191-195.

[13] C. Hernando, M. Mora, I. M. Pelayo, C. Seara and D. R. Wood, Extremal graph theory for metric dimension and diameter, Electron. J. Combin. 17 (2010), no. 1, Research Paper 30, 28 pp.

[14] D. L. Johnson, Topics in the Theory of Group Presentations, London Mathematical Society Lecture Note Series 42, Cambridge University Press, Cambridge, New York, 1980 .

[15] A. V. Kelarev, Ring Constructions and Applications, Series in Algebra 9, World Scientific, River Edge, NJ, 2002.

[16] Graph Algebras and Automata, Monographs and Textbooks in Pure and Applied Mathematics 257, Marcel Dekker, New York, 2003.

[17] _ Labelled Cayley graphs and minimal automata, Australas. J. Combin. 30 (2004), 95-101.

[18] A. V. Kelarev and S. J. Quinn, A combinatorial property and power graphs of groups, in: Contributions to General Algebra 12 (Vienna, 1999), 229-235, Heyn, Klagenfurt, 2000 .

[19] A. V. Kelarev, J. Ryan and J. Yearwood, Cayley graphs as classifiers for data mining: The influence of asymmetries, Discret Math. 309 (2009), no. 17, 5360-5369.

[20] X. Ma, Proper connection of power graphs of finite groups, J. Algebra Appl. 20 (2021), no. 3, Paper No. 2150033, 14 pp.

[21] X. Ma, G. L. Walls and K. Wang, Power graphs of (non)orientable genus two, Comm. Algebra 47 (2019), no. 1, 276-288.

[22] R. P. Panda, A combinatorial characterization of finite groups of prime exponent, Indag. Math. (N.S.) 31 (2020), no. 1, 1-6.

[23] R. Rajkumar and T. Anitha, Reduced power graph of a group, in: International Conference on Current Trends in Graph Theory and Computation, 69-76, Electron. Notes Discrete Math. 63, Elsevier Sci. B. V., Amsterdam, 2017. 
[24] _ Laplacian spectrum of reduced power graph of certain finite groups, Linear Multilinear Algebra 69 (2021), no. 9, 1716-1733.

[25] _ Some results on the reduced power graph of a group, Southeast Asian Bull. Math. 45 (2021), no. 2, 241-262.

[26] P. J. Slater, Leaves of trees, Congr. Numer. 14 (1975), 549-559.

Xuanlong Ma and Lan Li

School of Science, Xi'an Shiyou University, Xi'an 710065, China

E-mail addresses: xuanlma@mail.bnu.edu.cn, lanli98@126.com 